\title{
Resultados de la vigilancia de susceptibilidad antimicrobiana en Chile: Consolidando una red
}

\author{
Francisco Silva O., Marcela Cifuentes D. y M. Eugenia Pinto C., \\ en representación del Grupo Colaborativo de Resistencia Antimicrobiana
}

\section{Results of antimicrobial susceptibility surveillance in Chile: Consolidating a network}

Antimicrobial resistance is an increasing public health issue. Creation of surveillance networks is a matter of major importance for antimicrobial resistance understanding and monitoring. Also, they contribute to design actions for controlling its appearance and dissemination. In 2007 a colaborative group in antimicrobial resistance was formed, representing several health institutions across the country. In this paper we report the results of 2009 surveillance with emphasis in its interpretation, limitations and future perspectives.

Key words: Drug resistance, bacterial, anti infective agents, epidemiology.

Palabras clave: Resistencia bacteriana, antimicrobianos, epidemiología.

\section{Introducción}

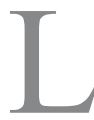

a emergencia de resistencia a antimicrobianos constituye un problema real en la atención de salud $^{1,2}$. Hay evidencias de cambios epidemiológicos significativos en los últimos 25 años en relación a las infecciones adquiridas en el ámbito hospitalario y en la comunidad. Entre los cambios de mayor impacto destaca el incremento de la resistencia ${ }^{3}$. Por ello, la Organización Mundial de la Salud ha enfatizado la necesidad de implementar, entre otros, programas de vigilancia y programas de intervención, que puedan traducirse en la reducción de la mortalidad asociada a infecciones, la morbilidad y los costos socioeconómicos que implica la resistencia a antimicrobianos ${ }^{4}$.

Se sabe que los esfuerzos orientados a su control se refieren, muy especialmente, a la prevención y control de infecciones, al uso prudente de los antimicrobianos y, en forma muy importante, a la vigilancia de la resistencia ${ }^{1}$. Esta vigilancia permite obtener la información necesaria para conocer la magnitud de los problemas en una determinada región y evaluar posteriormente el impacto global de las intervenciones que se proponen y que están orientadas a su reducción y control ${ }^{5}$.

La obtención de datos cuantitativos de resistencia en microorganismos considerados claves por su prevalencia y/o por sus particulares mecanismos de resistencia, es considerada un factor fundamental en la comprensión del fenómeno, favoreciendo el diseño de estrategias destinadas a prevenir la emergencia de nuevas resistencias y su posterior diseminación ${ }^{1,6-8}$. Estos estudios de vigilancia permiten además establecer las tendencias en un patógeno determinado y, en especial, reconocer problemas emergentes en los microorganismos vigilados ${ }^{9}$.

A ello se agrega que los datos obtenidos pueden ser comparados en períodos de tiempo sucesivos, permitiendo conocer la dinámica del proceso en una determinada zona geográfica y en un grupo de hospitales representativos ${ }^{6}$. También el conocimiento y difusión de los hallazgos referidos a la distribución de los perfiles de resistencia en las infecciones más frecuentes, puede favorecer las recomendaciones para un uso adecuado de antimicrobianos $^{1}$. Estos programas de vigilancia continua que estudian cepas representativas de diferentes áreas geográficas, son necesarios para conocer datos en tiempo real y su objetivo es introducir medidas efectivas de control que respondan a la información objetivada.

Se reconoce que la recolección sistematizada de información, el análisis e interpretación de los datos, facilitarán la planificación e implementación de programas, la necesaria difusión de los datos obtenidos y la posterior evaluación de su impacto en la práctica clínica y en salud pública ${ }^{1}$.

En este contexto, desde el año 2007 un grupo de microbiólogos e infectólogos de hospitales públicos, institucionales, universitarios y clínicas privadas se ha reunido periódicamente para establecer los lineamientos básicos de una red de vigilancia de susceptibilidad antimicrobiana, de alcance nacional, que pueda producir datos de manera estandarizada, mantenida y comparable en el tiempo.
Hospital Clínico Universidad de Chile, Santiago (FSO, MEPC) Hospital San Borja Arriarán, Santiago (MCD)

Conflictos de interés. Los autores declaran que han recibido apoyo logístico de MSD Chile para la organización de las reuniones de coordinación del grupo colaborativo. MSD no ha tenido influencia sobre las decisiones del método de vigilancia, selección de los participantes del grupo, generación o análisis de los resultados. Los autores no han recibido remuneraciones de MSD u otra institución por su participación en el grupo colaborativo.

Recibido: 7 de enero 2011

Correspondencia a: Francisco Silva Ojeda fsilva@redclinicauchile.cl 
A partir de ello, se cuenta con datos desde al año 2007 a la fecha, siendo el 2008 y 2009, años en que se avanzó hacia la consolidación de la red.

En el presente artículo, se presentan los datos consolidados de los establecimientos que aportaron la información del año 2009 como una primera aproximación a la difusión de estos contenidos (Tabla 1).

\section{Materiales y Métodos}

Se utilizó un sistema de recopilación de datos similar al sugerido por el Clinical and Laboratory Standards Institute (CLSI) ${ }^{10,11}$ donde los laboratorios de microbiología recolectaron su información local y la enviaron en planilla estándares iguales para todos los centros y luego se centralizó la consolidación de los mismos en una única base de datos general.

Se estableció una vigilancia anual, informando números (no porcentajes) de cepas totales probadas para cada combinación antimicrobiano/microorganismo y número de cepas susceptibles. Se indicó que las cepas a seleccionar debían ser: sólo una cepa por especie por paciente -la primera en caso de que tuviera varios aislados-, diferenciado por procedencia (ambulatorio, hospitalizado), de algunos sitios como sangre, orina o todos juntos. Además, sólo debían ser incluidas cepas

Tabla 1. Centros Informantes de datos de susceptibilidad in vitro para el año 2009

Centros Informantes 2009*

Hospital Regional de Iquique

Hospital Naval "Almirante Nef"

Hospital Roberto del Río

Hospital Clínico Universidad de Chile

Hospital de la Fuerza Aérea "Dr. Raúl Yazigi"

Hospital Dipreca

Hospital Militar de Santiago

Hospital del Salvador

Hospital Luis Calvo Mackenna

Integramédica

Hospital Clínico Universidad Católica

Hospital San Borja Arriarán

Hospital Higueras de Talcahuano

Hospital Regional de Concepción

*Se debe destacar, además, la participación de otros centros en la recolección de datos del año 2008: H. San Juan de Dios, H. Gustavo Fricke, Clínica Las Condes y, del año 2007: H. Sótero del Río, H. San José y H. Barros Luco Trudeau. provenientes de muestras para diagnóstico clínico y no para vigilancia epidemiológica.

Los datos se obtuvieron a través de diversas formas, yendo desde lo manual (contando cada aislado) a sistemas informáticos propios de la institución, programa Whonet (OMS, www.whonet.org), Kernmic ${ }^{\circledR}$ y BactLab ${ }^{\circledR}$.

Para el total de cepas reportadas por cada hospital, se calculó los porcentajes de susceptibilidad frente a cada agente antimicrobiano. Los resultados se expresan como un conglomerado de todos los centros, manteniéndose anónimos los resultados de cada centro en particular.

Los centros mantuvieron sus respectivas políticas de obtención de muestra y procedimientos de cultivos y antibiogramas. No se intervinieron ni supervisaron los procedimientos de control de calidad o el sistema de registro de los resultados de pacientes individuales.

Los microorganismos a vigilar se seleccionaron en forma consensuada en reunión con la mayoría de los participantes el año 2007. Estos son: Escherichia coli, Klebsiella pneumoniae, Pseudomonas aeruginosa, Acinetobacter baumannii, Enterococcus faecalis, Enterococcus faecium, Enterococcus spp y Streptococcus pneumoniae, agregándose en el año 2008, Enterobacter cloacae y Staphylococcus aureus. Para cada microorganismo se solicitó informar ciertos tipos de muestras (sangre, orina o sin diferenciación) y categorizarlos según origen (pediátrico o adulto) y procedencia (ambulatorio, unidad de paciente crítico, servicio hospitalizado no crítico o sin diferenciación de servicio) (Tabla 2).

\section{Resultados}

Centros participantes: Del total de centros que participó en el grupo colaborativo, enviaron datos para el período 2009, 14 centros. En conjunto, estos representan un total de 4.711 camas de pacientes, correspondiendo a camas críticas 528 de ellas. Los centros se distribuyeron en las regiones de Tarapacá $\left(\mathrm{I}^{\mathrm{a}}\right)$, Valparaíso $\left(\mathrm{V}^{\mathrm{a}}\right)$, Metropolitana $\left(\mathrm{XIII}^{\mathrm{a}}\right)$ y Bío-Bío $\left(\mathrm{VIII}^{\mathrm{a}}\right)$.

\section{Resultados acumulados de susceptibilidad}

Escherichia coli. En las Tabla 3 y 4 se muestra la susceptibilidad in vitro de las cepas de E. coli aisladas desde urocultivos de pacientes hospitalizados y ambulatorios, tanto en niños como en adultos. Lo mismo para cepas aisladas desde hemocultivos.

En el caso de las cepas urinarias de adultos destaca la baja susceptibilidad a ampicilina y cotrimoxazol, la que es significativamente menor en cepas de pacientes hospitalizados. Si bien para cefalotina la susceptibilidad en cepas de pacientes ambulatorios alcanza a $68,2 \%$, en 16.112 cepas estudiadas existe $22,2 \%$ de cepas intermedias a cefalotina y sólo $10 \%$ de cepas resistentes. Este 
dato puede resultar interesante especialmente en aislados provenientes de vía urinaria. Aunque no se solicitó el porcentaje de $\beta$-lactamasas de espectro extendido ( $\beta \mathrm{LEE}$ ) presente en las cepas informadas, se observa que 5,6 y $21 \%$ de las cepas urinarias de pacientes ambulatorios y hospitalizados, respectivamente, no son susceptibles a cefalosporinas de $3^{\text {a }}$ generación.

Klebsiella pneumoniae. En la Tabla 5 se muestran los resultados de susceptibilidad in vitro de $K$. pneumoniae frente a carbapenémicos. Destaca un porcentaje entre 90 y $93 \%$ de cepas susceptibles a ertapenem, dependiendo de la procedencia. Esta susceptibilidad es muy variada entre los centro, con un rango entre 44 y $100 \%$ en unidad de paciente crítico (UPC) y entre 64 y $99 \%$ en unidades no críticas.

Enterobacter cloacae. En la Tabla 7 se muestran los resultados de susceptibilidad in vitro a carbapenémicos. Sólo se cuenta con datos de adultos ya que no hay cepas suficientes para análisis en pacientes pediátricos. Destaca que en ertapenem sólo existe $\sim 80 \%$ de susceptibilidad pero, igual que en los casos anteriores, hay que destacar su variabilidad entre los distintos centros, con un rango entre 71 y $100 \%$ de cepas susceptibles y que además no existe una concordancia total entre el número de cepas testeadas para ertapenem, imipenem y meropenem.

Pseudomonas aeruginosa. En este caso destaca que para ninguno de los antimicrobianos, salvo colistín, el porcentaje de cepas susceptibles supera el $80 \%$. En particular, en el caso de los carbapenémicos en pacientes hospitalizados, la susceptibilidad es variable entre los dis-

\begin{tabular}{|c|c|c|}
\hline Agente & Muestra & Procedencia \\
\hline Escherichia coli & $\begin{array}{l}\text { Orina (urocultivos) } \\
\text { Sangre (hemocultivos) }\end{array}$ & $\begin{array}{l}\text { Ambulatoria } \\
\text { Hospitalizados } \\
\text { Independiente de la procedencia }\end{array}$ \\
\hline Klebsiella pneumoniae & Cualquier tipo & $\begin{array}{l}\text { Unidades de paciente crítico (UPC) } \\
\text { Servicios no crítico (no UPC) } \\
\text { Hospitalizados en general }\end{array}$ \\
\hline Enterobacter cloacae & Cualquier tipo & $\begin{array}{l}\text { Unidades de paciente crítico (UPC) } \\
\text { Servicios no críticos (no UPC) } \\
\text { Hospitalizados en general }\end{array}$ \\
\hline Pseudomonas aeruginosa & Cualquier tipo & $\begin{array}{l}\text { Unidades de paciente crítico (UPC) } \\
\text { Servicios no críticos (no UPC) } \\
\text { Hospitalizados en general }\end{array}$ \\
\hline Acinetobacter baumannii & Cualquier tipo & $\begin{array}{l}\text { Unidades de paciente crítico (UPC) } \\
\text { Servicios no críticos (no UPC) } \\
\text { Hospitalizados en general }\end{array}$ \\
\hline Staphylococcus aureus & Cualquier tipo & $\begin{array}{l}\text { Ambulatorio } \\
\text { Unidades de paciente crítico (UPC) } \\
\text { Servicios no críticos (no UPC) } \\
\text { Hospitalizados en general }\end{array}$ \\
\hline Enterococcus faecium & Cualquier tipo & $\begin{array}{l}\text { Unidades de paciente crítico (UPC) } \\
\text { Servicios no críticos (no UPC) } \\
\text { Hospitalizados en general }\end{array}$ \\
\hline Enterococcus faecalis & Cualquier tipo & $\begin{array}{l}\text { Unidades de paciente crítico (UPC) } \\
\text { Servicios no críticos (no UPC) } \\
\text { Hospitalizados en general }\end{array}$ \\
\hline Streptococcus pneumoniae & $\begin{array}{l}\text { Sangre (hemocultivos), líquido } \\
\text { cefalorraquídeo y otros líqui- } \\
\text { dos estériles }\end{array}$ & Independiente de la procedencia \\
\hline
\end{tabular}

Tabla 3. Susceptibilidad in vitro de Escherichia coli año 2009 en población adulta. Los datos están expresados en porcentaje de cepas susceptibles

\begin{tabular}{|c|c|c|c|}
\hline Antimicrobiano & $\begin{array}{c}\text { Urocultivos } \\
\text { ambulatorios } \\
(\mathrm{N}: 36.949) \\
\%\end{array}$ & $\begin{array}{c}\text { Urocultivos } \\
\text { hospitalizados } \\
\text { (N: } 4.588) \\
\%\end{array}$ & $\begin{array}{c}\text { Hemocultivos } \\
\qquad \begin{array}{c}(\mathrm{N}: 465) \\
\%\end{array}\end{array}$ \\
\hline Gentamicina & 93,1 & 84,5 & 77,9 \\
\hline Amikacina & $92,5^{*}$ & 95,8 & 95,4 \\
\hline Ciprofloxacina & 80,4 & 64,4 & 60,9 \\
\hline Nitrofurantoína & 96,0 & 90,9 & - \\
\hline Cotrimoxazol & 70,4 & 63,1 & 61,0 \\
\hline Ampicilina & 46,4 & 29,7 & 32,1 \\
\hline Cefalotina & 68,2 & 55,1 & 50,0 \\
\hline Cefotaxima & 94,4 & 79,0 & 77,8 \\
\hline Piperacilina/tazobactam & 93,6 & 91,0 & 90,3 \\
\hline Ertapenem & 100,0 & 99,9 & 100,0 \\
\hline Imipenem & 100,0 & 100,0 & 100,0 \\
\hline Meropenem & 100,0 & 100,0 & 100,0 \\
\hline
\end{tabular}

Tabla 4. Susceptibilidad in vitro de Escherichia coli año 2009 en población pediátrica. Los datos están expresados en porcentaje de cepas susceptibles

\begin{tabular}{|lccc|}
\hline Antimicrobiano & $\begin{array}{c}\text { Urocultivos } \\
\text { ambulatorios } \\
(\mathrm{N}: 2.556) \\
\%\end{array}$ & $\begin{array}{c}\text { Urocultivos } \\
\text { hospitalizados } \\
(\mathrm{N}: 302) \\
\%\end{array}$ & Hemocultivos \\
\hline Gentamicina & 95,9 & 93,2 & $\%$ \\
Amikacina & 99,5 & 90,4 & 95,1 \\
\hline Ciprofloxacina & 94,9 & 73,6 & 93,6 \\
\hline Nitrofurantoína & 98,1 & 93,5 & 85,9 \\
\hline Cotrimoxazol & 71,5 & 53,1 & - \\
Ampicilina & 41,7 & 29,4 & 52,5 \\
\hline Cefalotina & 67,9 & 60,2 & 35,7 \\
Cefotaxima & 97,3 & 80,3 & 62,5 \\
\hline Ertapenem & 100,0 & 100,0 & 83,1 \\
Imipenem & 100,0 & 100,0 & 100,0 \\
\hline Meropenem & 100,0 & 100,0 & 100,0 \\
\hline
\end{tabular}


tintos centros con porcentajes que van entre 55 y $100 \%$ para imipenem, 45 y $100 \%$ para meropenem. En el caso de la susceptibilidad a colistín, sólo se tiene información de 19\% de las cepas estudiadas, probablemente las más resistentes a otros agentes de primera y segunda línea, por lo que el 93,6\% de susceptibilidad debe ser interpretado dentro de dicho contexto (Tabla 8). Distinto es el panorama de susceptibilidad de las cepas aisladas de niños donde se muestra una mejor sensibilidad a todos los antimicrobianos (Tabla 9).

Acinetobacter baumannii. En la Tabla 10 se observa que para todos los antimicrobianos de primera línea el porcentaje de susceptibilidad in vitro en pacientes hospitalizados es inferior a $70 \%$. En el caso de imipenem, destaca la amplia variabilidad entre los centros con rangos entre 22 y $100 \%$. Lo mismo ocurre con meropenem (rangos entre 18 y $100 \%$ de susceptibilidad). En los antimicrobianos de última línea como

Tabla 5. Susceptibilidad in vitro de Klebsiella pneumoniae año 2009 en población adulta. Los datos están expresados en porcentaje de cepas susceptibles

\begin{tabular}{|lccc|}
\hline Antimicrobiano & $\begin{array}{c}\text { Hospitalizados } \\
(\mathrm{N}: 2.118)\end{array}$ & $\begin{array}{c}\text { UPC } \\
(\mathrm{N}: 592)\end{array}$ & $\begin{array}{c}\text { No UPC } \\
(\mathrm{N}: 1.348)\end{array}$ \\
\hline Ertapenem & $\begin{array}{c}\% \\
\text { Imipenem }\end{array}$ & 90,4 & 9 \\
Meropenem & 97,5 & 98,6 & 96,9 \\
\hline UPC: unidad de pacientes crítico & 98,1 & 96,2 & 99,0 \\
\hline
\end{tabular}

Tabla 6. Susceptibilidad in vitro de Klebsiella pneumoniae año 2009 en población pediátrica. Los datos están expresados en porcentaje de cepas susceptibles

\begin{tabular}{|lcc|}
\hline Antimicrobiano & $\begin{array}{c}\text { Hospitalizados } \\
(\mathrm{N}: 200)\end{array}$ & $\begin{array}{c}\text { UPC } \\
(\mathrm{N}: 61)\end{array}$ \\
Ertapenem & $\%$ & $\%$ \\
Imipenem & 100,0 & 100,0 \\
Meropenem & 97,9 & 100,0 \\
\hline
\end{tabular}

UPC: unidad de paciente crítico

Tabla 7. Susceptibilidad in vitro de Enterobacter cloacae año 2009 en población adulta. Los datos están expresados en porcentaje de cepas susceptibles

\begin{tabular}{|c|c|c|c|}
\hline Antimicrobiano & $\begin{array}{c}\text { Hospitalizados } \\
\text { (N: } 462) \\
\%\end{array}$ & $\begin{array}{c}\text { UPC } \\
(\mathrm{N}: 87) \\
\%\end{array}$ & $\begin{array}{c}\text { No UPC } \\
\text { (N: 289) } \\
\%\end{array}$ \\
\hline Ertapenem & 80,7 & 83,3 & 80,1 \\
\hline Imipenem & 99,4 & 98,7 & 100,0 \\
\hline Meropenem & 99,0 & 97,2 & 99,3 \\
\hline
\end{tabular}

Tabla 8. Susceptibilidad in vitro de Pseudomonas aeruginosa año 2009 en población adulta. Los datos están expresados en porcentaje de cepas susceptibles

\begin{tabular}{|lccc|} 
Antimicrobiano & $\begin{array}{c}\text { Hospitalizados } \\
(\mathrm{N}: 1.935) \\
\%\end{array}$ & $\begin{array}{c}\text { UPC } \\
\text { (N: } 716) \\
\%\end{array}$ & $\begin{array}{c}\text { No UPC } \\
\text { (N: } 967) \\
\%\end{array}$ \\
Gentamicina & 66,5 & 62,5 & 65,9 \\
Amikacina & 78,8 & 75,5 & 77,7 \\
Ciprofloxacina & 51,5 & 46,0 & 51,0 \\
Ceftazidima & 63,2 & 56,2 & 65,2 \\
Cefepime & 46,3 & 33,6 & 45,9 \\
Piperacilina/tazobactam & 63,3 & 57,4 & 61,2 \\
Imipenem & 68,4 & 53,7 & 78,0 \\
Meropenem & 69,8 & 57,7 & 76,7 \\
Colistín & 95,3 & 93,6 & 93,6 \\
\hline UPC: unidad de paciente crítico & & \\
\hline
\end{tabular}

Tabla 9. Susceptibilidad in vitro de Pseudomonas aeruginosa año 2009 en población pediátrica. Los datos están expresados en porcentaje de cepas susceptibles

\begin{tabular}{|lcc|}
\hline Antimicrobiano & $\begin{array}{c}\text { Hospitalizados } \\
(\mathrm{N}: 177) \\
\%\end{array}$ & $\begin{array}{c}\text { UPC } \\
(\mathrm{N}: 65) \\
\%\end{array}$ \\
Gentamicina & 76,7 & 92,2 \\
Amikacina & 83,1 & 93,8 \\
Ciprofloxacina & 80,8 & 90,8 \\
Ceftazidima & 89,8 & 76,6 \\
Cefepime & 88,6 & 84,4 \\
Imipenem & 91,5 & 81,5 \\
Meropenem & 90,7 & 78,3 \\
\hline UPC: unidad de paciente crítico & & \\
\hline
\end{tabular}

Tabla 10. Susceptibilidad in vitro de Acinetobacter baumannif año 2009 en población adulta. Los datos están expresados en porcentaje de cepas susceptibles

\begin{tabular}{|lccc|}
\hline Antimicrobiano & $\begin{array}{c}\text { Hospitalizados } \\
(\mathrm{N}: 840) \\
\%\end{array}$ & $\begin{array}{c}\text { UPC } \\
(\mathrm{N}: 454)\end{array}$ & $\begin{array}{c}\text { No UPC } \\
\text { (N: 324) }\end{array}$ \\
Gentamicina & 59,3 & 55,5 & $\%$ \\
Amikacina & 41,4 & 35,0 & 65,0 \\
Ciprofloxacina & 21,7 & 19,8 & 48,9 \\
Ampicilina/sulbactam & 63,9 & 59,0 & 25,1 \\
Imipenem & 70,1 & 62,4 & 72,2 \\
Meropenem & 67,6 & 57,3 & 82,8 \\
Colistín & 100,0 & 100,0 & 84,2 \\
\hline Tigeciclina & 94,8 & - & 100,0 \\
\hline
\end{tabular}


colistín, se evidencia $100 \%$ de susceptibilidad, pero en este caso sólo se cuenta con información de $15 \%$ de las cepas. Para tigeciclina se tiene información de $25 \%$ de las cepas totales, lo que sumado a la variabilidad de los métodos de estudio utilizados (dilución en agar, difusión

Tabla 11. Susceptibilidad in vitro de Acinetobacter baumanni año 2009 en población pediátrica. Los datos están expresados en porcentaje de cepas susceptibles

\begin{tabular}{|lc|}
\hline Antimicrobiano & $\begin{array}{c}\text { Hospitalizados } \\
(\mathrm{N}: 62) \\
\%\end{array}$ \\
\hline Gentamicina & 89,5 \\
Amikacina & 62,7 \\
Ciprofloxacina & 53,2 \\
Ampicilina/sulbactam & 72,0 \\
Imipenem & 91,9 \\
Meropenem & 91,9 \\
\hline
\end{tabular}

con disco, epsilometría y automatizados) hace que el 94\% de susceptibilidad deba ser analizado con precaución.

En niños no fue posible obtener datos de susceptibilidad in vitro de $A$. baumannii diferenciados entre pacientes críticos y no críticos por el bajo número de cepas. En la Tabla 11 se muestras los datos de pacientes hospitalizados, en forma independiente de la localización de la infección.

Staphylococcus aureus. En las Tablas 12 y 13 se muestran los datos de susceptibilidad in vitro de $S$. aureus en adultos y niños. En las cepas aisladas de adultos destaca la elevada resistencia a meticilina (cloxacilina) en UPC, con susceptibilidades a cloxacilina que van entre 14 y $58 \%$.

En los pacientes ambulatorios adultos se observa una susceptibilidad a cloxacilina de $82 \%$ con rangos entre 50 y $97 \%$. Cabe recordar que estos datos se refieren a cepas que fueron obtenidas de cultivos tomados a los pacientes de manera ambulatoria, pero que no necesariamente son cepas adquiridas en la comunidad.

En ninguno de los casos, adultos o niños, hospitalizados o ambulatorios se detectó la presencia de cepas no susceptibles a vancomicina

Tabla 12. Susceptibilidad in vitro de Staphylococcus aureus año 2009 en población adulta. Los datos están expresados en porcentaje de cepas susceptibles

\begin{tabular}{|lcccc|}
\hline Antimicrobiano & $\begin{array}{c}\text { Ambulatorios } \\
(\mathrm{N}: 939)\end{array}$ & $\begin{array}{c}\text { UPC } \\
(\mathrm{N}: 984)\end{array}$ & $\begin{array}{c}\text { No UPC } \\
(\mathrm{N}: 2.354)\end{array}$ & $\begin{array}{c}\text { Hospitalizados } \\
\text { (N: 3.536) } \\
\%\end{array}$ \\
Cloxacilina & $\%$ & $\%$ & $4 \%$ & 42,0 \\
Vancomicina & 82,3 & 23,5 & 47,6 & 100,0 \\
Cotrimoxazol & 100,0 & 100,0 & 100,0 & 88,9 \\
Rifampicina & 97,2 & 87,2 & 88,8 & 92,5 \\
\hline Eritromicina & 98,0 & 94,6 & 90,9 & 34,9 \\
Clindamicina & 72,6 & 21,1 & 40,0 & 38,1 \\
\hline UPC: unidad de paciente crítico & 78,5 & 22,2 & 42,7 & \\
\hline
\end{tabular}

Tabla 13. Susceptibilidad in vitro de Staphylococcus aureus año 2009 en población pediátrica. Los datos están expresados en porcentaje de cepas susceptibles. No se muestran resultados de susceptibilidad a eritromicina ya que no es probada en todos los centros

\begin{tabular}{|c|c|c|c|c|}
\hline Antimicrobiano & $\begin{array}{c}\text { Ambulatorios } \\
\text { (N: } 145) \\
\%\end{array}$ & $\begin{array}{c}\text { UPC } \\
\text { (N: 122) } \\
\%\end{array}$ & $\begin{array}{c}\text { No UPC } \\
(\mathrm{N}: 178) \\
\%\end{array}$ & $\begin{array}{c}\text { Hospitalizados } \\
\text { (N: 222) } \\
\%\end{array}$ \\
\hline Cloxacilina & 93,1 & 70,0 & 55,1 & 55,9 \\
\hline Vancomicina & 100,0 & 100,0 & 100,0 & 100,0 \\
\hline Cotrimoxazol & 99,3 & 94,2 & 87,0 & 87,3 \\
\hline Rifampicina & 97,2 & 98,3 & 95,5 & 95,5 \\
\hline Clindamicina & 84,1 & 64,2 & 61,6 & 59,6 \\
\hline
\end{tabular}


Tabla 14. Susceptibilidad in vitro de Enterococcus faecium año 2009 en población adulta. Los datos están expresados en porcentaje de cepas susceptibles

\begin{tabular}{|lccc|} 
Antimicrobiano & $\begin{array}{c}\text { Hospitalizados } \\
(\mathrm{N}: 807)\end{array}$ & $\begin{array}{c}\text { UPC } \\
(\mathrm{N}: 263)\end{array}$ & $\begin{array}{c}\text { No UPC } \\
(\mathrm{N}: 404) \\
\%\end{array}$ \\
$\begin{array}{l}\% \\
\text { Ampicilina }\end{array}$ & 6,4 & 3,2 & $\%$ \\
Vancomicina & 31,1 & 14,2 & 7,2 \\
\hline Teicoplanina & $59,8^{*}$ & $61,0^{*}$ & 38,4 \\
\hline
\end{tabular}

UPC: unidad de paciente crítico. *Se cuenta con información de susceptibilidad a teicoplanina solamente del $50 \%$ de las cepas totales.

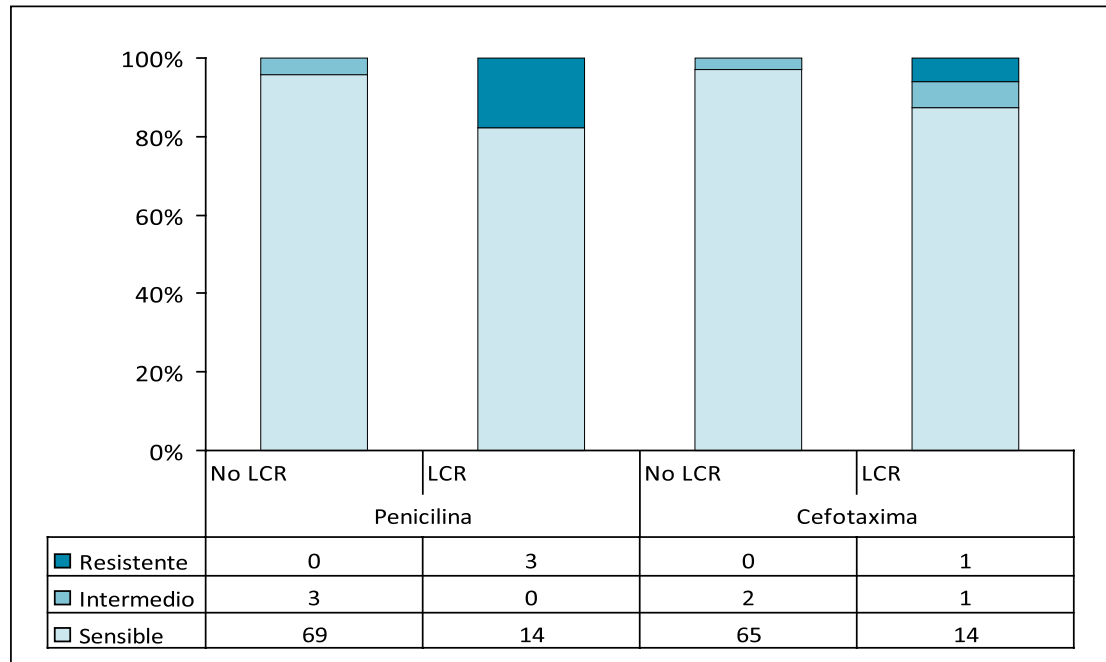

Figura 1. Susceptibilidad in vitro de Streptococcus pneumoniae aislado de muestras de líquido cefalorraquídeo (LCR) y otras muestras de líquidos estériles (no LCR) en población pediátrica. Año 2009.

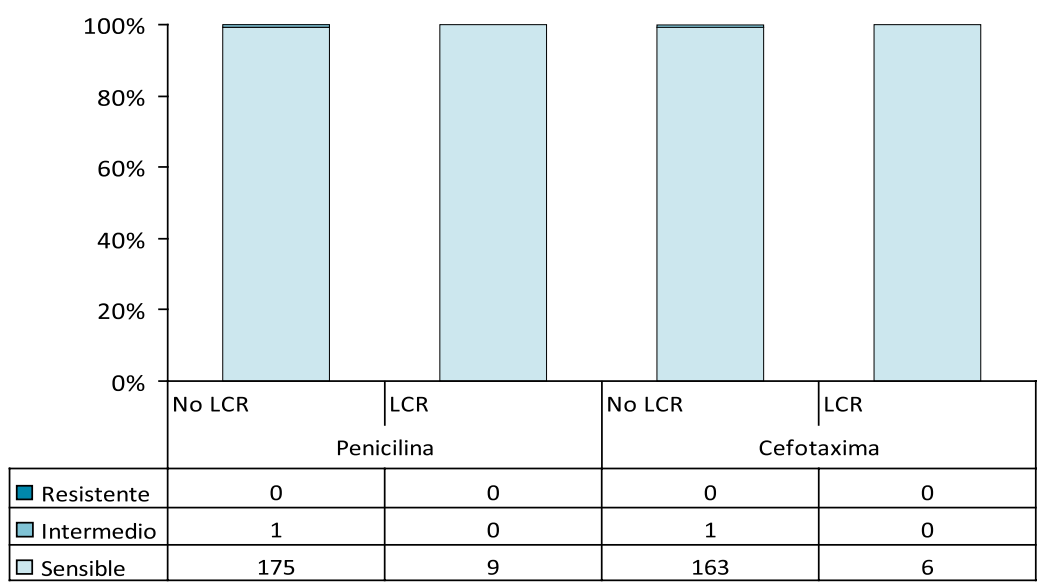

Figura 2. Susceptibilidad in vitro de Streptococcus pneumoniae aislado de muestras de líquido cefalorraquídeo (LCR) y otras muestras de líquidos estériles (no LCR) en población adulta. Año 2009.
Enterococcus spp. En la Tabla 14 se muestran los datos de susceptibilidad in vitro de E. faecium en adultos, destacando una susceptibilidad de $14 \%$ a vancomicina en cepas aisladas en unidades de pacientes críticos. La presencia de cepas no susceptibles a teicoplanina nos indica la presencia del fenotipo vanA (el porcentaje de susceptibilidad a teicoplanina está calculado solamente sobre $50 \%$ de las cepas totales).

En E. faecalis se recibió información de 1.910 cepas de pacientes hospitalizados, encontrándose sólo 16 cepas resistentes a vancomicina ( $98,9 \%$ de cepas susceptibles)

No se calculó la susceptibilidad in vitro de Enterococcus spp en niños por el bajo número de aislados.

Streptococcus pneumoniae. En las Figuras 1 y 2, se muestra que, tanto en la población pediátrica como en la de adultos, la sensibilidad a penicilina y a cefotaxima es de casi $100 \%$ encontrándose cepas resistentes sólo en los aislados de muestras provenientes de niños, específicamente en LCR, donde tanto penicilina como cefotaxima tienen algún grado de resistencia, en un porcentaje bajo pero no despreciable.

\section{Comentarios}

Estos datos constituyen los primeros resultados de un grupo colaborativo, con alcances nacionales, que se ha mantenido estable desde el año 2007. Si bien tienen limitaciones que serán discutidas más adelante, hay puntos a considerar en el análisis de los perfiles de susceptibilidad de cada agente en particular.

Para E. coli destaca que, en todas las muestras informadas se mantiene una buena susceptibilidad a aminoglucósidos, pese al prolongado tiempo de uso. No ocurre lo mismo con cefalosporinas de $1^{\mathrm{a}}$ a $3^{\mathrm{a}}$ generación en que se observa una susceptibilidad variable pero menor a la anterior. En este mismo sentido, destaca la presencia de resistencia a cefalosporinas de $3^{\circ}$ generación en pacientes ambulatorios, que, al igual que en el caso de las cepas hospitalarias, se asocia, en la mayoría de los casos, probablemente a presencia de $\beta$ LEE. Estos datos deberían llevar a cuestionarse los tratamientos empíricos iniciales de pacientes con sospecha de infección por E. coli.

Para el caso de $K$. pneumoniae y E. cloacae, se decidió vigilar sólo la susceptibilidad in vitro a carbapenémicos ya que está bien documentada la resistencia a cefalosporinas de $3^{\text {a }}$ generación mediada por $\beta$ LEE o enzimas tipo AmpC, respectivamente ${ }^{12}$. En ambos casos se observa la aparición de cepas no susceptibles a carbapenémicos, en especial a ertapenem, con dispersión en los porcentajes de susceptibilidad entre los distintos centros. Esta dispersión podría deberse a situaciones de hiperendemia o brotes no controlados en aquellos centros que tienen los menores porcentajes de susceptibilidad. En cuanto a los mecanismos de esta resistencia a carbapenémicos, de acuerdo a 
datos no publicados (Gonzalez G, 2010), indican que, a diferencia de lo que ocurre en algunos países donde la resistencia a carbapenémicos se debe a la presencia de enzimas tipo carbapenemasas, en nuestro país se debería a la presencia de enzimas tipo $\beta \mathrm{LEE}$ o AmpC, asociada a impermeabilidad por pérdida de porinas, por lo que se hace imprescindible mantener la vigilancia de estas cepas para detectar los primeros casos de resistencia por mecanismos enzimáticos transferibles. Por lo mismo, los ensayos fenotípicos de resistencia recomendados por CLSI para detección de carbapenemasas, en nuestra realidad, podrían dar falsos positivos y su indicación en forma masiva podría no ser recomendable, tal como ha ocurrido en Colombia (Villegas MV 2010, comunicación personal) y Argentina ${ }^{13,14}$.

En el caso de los no-fermentadores como $P$. aeruginosa y $A$. baumannii en población adulta, destacan los bajos porcentajes de susceptibilidad, relativamente homogéneos, a todos los antimicrobianos probados, lo que es reflejo del patrón de pan-resistencia reportado en los últimos años en múltiples hospitales de nuestro país; sin embargo, existen diferencias notorias entre los distintos hospitales (datos no mostrados) lo que podría deberse a la circulación de distintos clones y/o situaciones de brotes. Son excepciones a lo anterior, la susceptibilidad a colistín (P. aeruginosa y $A$. baumannii) y tigeciclina ( $A$. baumannii), antimicrobianos de última línea a los que se ha debido recurrir dada la falta de opciones terapéuticas. En estos casos, los datos de susceptibilidad deben ser analizados con precaución dado que la mayoría de los hospitales no los prueban de rutina, sino que solamente los estudian en los casos de cepas resistentes a los otros antimicrobianos, por lo que los datos podrían estar sesgados hacia una mayor resistencia.

En especies grampositivas se definió vigilar la susceptibilidad de $S$. aureus, Enterococcus spp y $S$. pneumoniae, dado la importancia de los dos primeros en infecciones nosocomiales y los problemas conocidos de resistencia que representan en su conjunto.

En $S$. aureus, se observa un alto porcentaje de resistencia a meticilina en pacientes hospitalizados, tanto en ambiente de UPC como no UPC, mientras que se ha mantenido una buena susceptibilidad a cotrimoxazol y rifampicina. Esto coincide con el tipo de clon de $S$. aureus resistente a meticilina (SARM) descrito como predominante en nuestro país ${ }^{15}$. En el caso de $S$. aureus aislados de pacientes ambulatorios destaca que alrededor de $20 \%$ de las cepas son no susceptibles a cloxacilina, lo cual no implica, necesariamente, la presencia de SARM adquirido en la comunidad (SARM-AC); en Chile se vigila rutinariamente y las sospechas de SARM-AC se derivan al Instituto de Salud Pública y se ha detectado que los reportes de estos casos son más bien escasos, comprobándose pocos de los sospechados. Esta resistencia probablemente está dada por pacientes ambulatorios que estuvieron hospitalizados en sus respectivos centros y que se han controlado incidentalmente en forma ambulatoria, tomándose muestras que finalmente el laboratorio detecta como muestras de procedencia ambulatoria a pesar de ser cepas de origen intrahospitalario. En el año 2009 se publicaron los datos de susceptibilidad de $S$. aureus de un centro exclusivamente ambulatorio donde se constata que la resistencia a cloxacilina no supera el 3\%. Este centro, también participa de nuestra red ${ }^{16}$.

En el caso de Enterococcus spp, si bien se solicitaron datos de E. faecium y E. faecalis, se observa que la resistencia a vancomicina se concentra en E. faecium, sobre todo en pacientes de UPC. Además se observa cerca de $40 \%$ de cepas con resistencia a teicoplanina lo que refleja la presencia del genotipo vanA que ha venido reportándose con mayor frecuencia desde el año 2007 y con un importante brote en un hospital de Santiago ese mismo año ${ }^{17,18}$.

Mención aparte merece el bajo porcentaje de resistencia de $S$. pneumoniae a penicilina en cepas de síndromes no meníngeos, lo que se contrapone a datos anteriores en que se observaban alta resistencia sobre todo en pacientes pediátricos ${ }^{19,20}$. Esta disminución no se debe solamente a cambios epidemiológicos sino a un importante cambio en los puntos de corte establecidos por el CLSI en el año 2008 (antes del 2008 se definía susceptibilidad con CIM de penicilina $\leq 0,06 \mu \mathrm{g} / \mathrm{ml}$ y desde el 2008 con $\mathrm{CIM} \leqq$ $2 \mu \mathrm{g} / \mathrm{ml})^{21}$.

Los estudios de vigilancia de resistencia tienen generalmente limitaciones y fuentes de sesgo reconocidas que se repiten ${ }^{1}$. Es así como la literatura médica muestra que las más importantes son:

- Errores en los denominadores (difícil diferenciación entre hospitalizado y comunitario).

- Los resultados son generales y no en condiciones ideales, (ej. los datos de E. coli en ITU no se refieren a primer episodio de ITU ni sólo a recurrencia sino a una proporción no determinada de estas).

- Diferencia en las prácticas de cultivos entre los centros.

- Inclusión de vigilancias epidemiológicas.

- El laboratorio no es capaz de diferenciar colonizaciones de infecciones.

- Inclusión sólo de hospitales de referencia.

- Conteo múltiple de cepas de un mismo paciente.

- Diferencia en los procedimientos de los laboratorios y control de calidad.

En el caso de nuestra evaluación podemos señalar que las limitaciones encontradas incluyen algunas de las ya mencionadas y además:

- Diferentes metodologías de determinación de susceptibilidad in vitro. 
- Variabilidad entre los antimicrobianos que prueba cada centro en sus antibiogramas primarios y complementarios.

- No se consideraron los ensayos fenotípicos de determinación de susceptibilidad a carbapenémicos (CLSI 2009).

- Diferente metodología de manejo de datos en los centros.

- No permite establecer prevalencia ni incidencia de los microorganismos en los hospitales ya que para ello debería calcularse según número de casos por días camas ocupadas $\mathrm{y} / \mathrm{o}$ egresos hospitalarios.

\section{Acciones futuras}

- Fortalecer el Grupo Colaborativo de Resistencia Antimicrobiana.

- Mantener la red de vigilancia de resistencia para establecer tendencias en el tiempo.

- Incorporar centros de regiones no representadas.

- Incorporar medida de incidencia y prevalencia de los microorganismos en infecciones de origen hospitalario (muestras de procedencia hospitalaria).

- Correlacionar con datos de infecciones nosocomiales y medidas de control y consumo de antimicrobianos de los mismos centros.

- Correlacionar los datos de susceptibilidad con el consumo de antimicrobianos medido mediante DDD.

- Detección precoz de nuevos patrones de resistencia y de mecanismos de resistencia.

En resumen, contener la diseminación de la resistencia a los antimicrobianos constituye uno de los mayores desafíos para el futuro. Numerosos datos muestran el impacto que significa en la reducción de la morbi-mortalidad y los costos asociados a las infecciones por microorganismos multi-resistentes.

Por lo tanto, el contar con datos confiables en frecuencia y tipos de resistencia detectadas en el país es de la mayor relevancia para la formulación de políticas en la elaboración de programas de prevención y control del problema. Esta información es generada por la vigilancia, la que debe continuar jugando un rol central en el monitoreo y seguimiento en el tiempo de aquellas resistencias ya establecidas y en la detección oportuna de aquellas consideradas emergentes.

\section{Colaboradores (en la generación y análisis de datos 2009)}

Luis Bavestrello F., Clínica Reñaca, Viña del Mar.

Helia Bello T., Universidad de Concepción, Concepción.

Dona Benadof F., Hospital Roberto del Río y Hospital Dipreca, Santiago.

Stepahnie Braun J., Hospital Militar de Santiago.
Isabel Briceño L., Hospital Naval “Almirante Nef”, Viña del Mar.

Rossanna Camponovo C., Integramédica, Santiago.

Palmira Choque, Hospital Regional de Iquique.

José Cofré G., Hospital Luis Calvo Mackenna, Santiago.

Erna Cona T., Hospital de la Fuerza Aérea "Dr. Raúl Yazigi".

Alejandra Fernández V., Hospital del Salvador, Santiago. Patricia García C., Hospital Clínico Universidad Católica. Gerardo González R., Universidad de Concepción, Concepción.

Chrystal Juliet L., Hospital del Salvador, Santiago.

Jaime Labarca L. Hospital Clínico Universidad Católica, Santiago.

Walter Ledermann D., Hospital Luis Calvo Mackenna, Santiago.

Olga López M., Hospital Regional de Iquique.

Lorena Porte T., Hospital Militar de Santiago y Hospital Clínico Universidad Católica.

Gisela Riedel M., Hospital Regional de Concepción, Concepción.

Pamela Rojas, Hospital Higueras de Talcahuano, Concepción.

Michel Serri V., Hospital de la Fuerza Aérea "Dr. Raúl Yazigi”, Santiago.

Agradecimientos. A todos los miembros del grupo colaborativo por su aporte en la discusión y mejoras en los datos aquí mostrados. No se citan todos los participantes desde el año 2007 a la fecha, sino que se reservó la autoría para los que hicieron posible la generación y entrega de resultados durante el año 2009. A MSD Chile por su apoyo en la organización y entrega de redes de contacto para las reuniones del grupo colaborativo. Además por las organización de todas las versiones del curso "Inmersión en resistencia bacteriana" en el cual se gestó la formación del Grupo de Resistencia Antimicrobiana.

\section{Resumen}

La resistencia antimicrobiana es un problema de salud pública en aumento. La generación de redes de vigilancia es una necesidad para la correcta comprensión y seguimiento de este fenómeno, además de ayudar a orientar las medidas para el control de su aparición y diseminación. En Chile, desde el año 2007 se constituyó un grupo colaborativo de resistencia antimicrobiana de centros de salud de varias regiones, que ha generado información respecto a la frecuencia de resistencia antimicrobiana de distintas especies bacterianas. En este artículo se presentan los resultados de la vigilancia del año 2009, con énfasis en su interpretación, limitaciones y perspectivas a futuro. 


\section{Referencias}

1.- Rempel O R, Laupland K B. Surveillance for antimicrobial resistant organisms: potential sources and magnitude of bias. Epidemiol Infect. 2009; 137 (12): 1665-73.

2.- Conly J. Antimicrobial resistance in Canada. CMAJ 2002; 167: 885-91.

3.- Colombo A L, Janini M, Salomao R, Medeiros E, Wey S B, Pignatari A C. Surveillance programs for detection and characterization of emergent pathogens and antimicrobial resistance. Results from the Division of Infectious Diseases, UNIFESP. An Acad Bras Cienc 2009; 81 (3): 571-87.

4.- Borg M A, Cookson B D, Zarb1 P, Scicluna E A. on behalf the ARMed Steering Group and Collaborators. Antibiotic resistance surveillance and control in the Mediterranean region: report of the ARMed Consensus Conference. J Infect Dev Ctries 2009; 3 (9): 654-9.

5.- Reynolds R. Antimicrobial resistance in the UK and Ireland. J Antimicrob Chemother 200; 64 (Suppl. 1), i19-i23.

6.- White A R, on behalf of the BSAC Working Parties on Resistance Surveillance. The British Society for Antimicrobial Chemotherapy Resistance Surveillance Project: a successful collaborative model. J Antimicrob Chemother 2008; 62 (Suppl 2): ii3-iil 14.

7.- Reynolds R, Hope R, Williams L, on behalf of the BSAC working parties on resistance surveillance. Survey, laboratory, and statistical methods for the BSAC Resistance Surveillance Programmes.

J Antimicrob Chemother 2008; 62 (Suppl 2): ii15-ii28.
8.- MacGowan A P, on behalf of the BSAC Working Parties on Resistance Surveillance. Clinical implications of antimicrobial resistance for therapy. J Antimicrob Chemother 2008; 62 (Suppl 2): ii 105-ii114.

9.- Masterton R. The importance and future of antimicrobial surveillance studies. Clin Infect Dis 2008; 47: S21-31.

10.- Hindler J, Stelling J. Analysis and presentation of cumulative antibiograms: a new consensus guideline from the Clinical and Laboratory Standards Institute. Clin Infect Dis 2007; 44: 867-73.

11.- Clinical and Laboratory Standards Institute (CLSI). Analysis and presentation of cumulative antimicrobial susceptibility test data. 2nd ed. Approved guideline M39-A2. Wayne, PA: CLSI, 2006

12.- Bello H, Trabal N, Ibáñez D, Reyes A, Domínguez M, Mella S, et al. Beta-lactamasas de familias diferentes a TEM y SHV en cepas de Klebsiella pneumoniae subespecie pneumoniae aisladas en hospitales chilenos. Rev Med Chile 2005; 133: 737-9.

13.- Pasteran F, Méndez T, Rapoport M, Guerriero L, Corso A. Controlling false-positive results obtained with the Hodge and Masuda assays for detection of class a carbapenemase in species of Enterobacteriaceae by incorporating boronic acid. J Clin Microbiol 2010; 48 (4): 1323-32.

14.- CLSI. Performance Standards for Antimicrobial Susceptibility Testing $9^{\text {th }}$ Informational Supplement. CLSI document M100-S19. Wayne PA: Clinical and Laboratoy Standards Institute; 2009.

15.- Rodríguez-Noriega E, Seas C, Guzmán-Blanco
M, Mejía C, Álvarez C, Bavestrello L, Zurita J, et al. Evolution of methicillin-resistant Staphylococcus aureus clones in Latin America. Int J Infect Dis 2010; 14 (7): e560-6.

16.- Camponovo R. Susceptibilidad bacteriana a antimicrobianos. Especies aisladas de pacientes ambulatorios de la Región Metropolitana, Chile, 2007. Rev Chil Infect 2009; 26 (1): 18-20.

17.- Fica A, Jemenao M I, Bilbao P, Ruiz G, Sakurada A, Pérez, Zúñiga I, et al. Emergencia de infecciones por Enterococcus sp resistente a vancomicina en un hospital universitario en Chile. Rev Chil Infectol 2007; 24 (6): 462-71

18.- Diomedi A, Blamey R, Juliet C, Fernández A, Garreaud C, Andrade A, et al. Un brote de Enterococcus faecium resistente a vancomicina, fenotipo Van A (ERV Van A) en el Hospital del Salvador. Descripción, estudio molecular, factores de riesgo y manejo epidemiológico. Libro de Resúmenes XIV Congreso Chileno de infectología. 2007, Pucón, Chile. Resumen CO 17.

19.- Soler T, Salamanca L, Arbo G, Molina E. In vitro susceptibility of Streptococcus pneumoniae strains isolated from lower respiratory infections in Instituto Nacional del Tórax (INT). Rev Med Chile 2002; 130(3): 304-8.

20.- Contreras L, Fica A, Figueroa O, Enríquez N, Urrutia P, Herrera P. Resistance to Streptococcus pneumoniae to penicillin and its association with clinical and epidemiological factors. Rev Med Chile 2002; 130 (1): 26-34.

21.- CLSI. Performance Standards for Antimicrobial Susceptibility testing eighteenth Informational Supplement. CLSI document M100-S18. Wayne PA: Clinical and Laboratoy Standards Institute; 2008. 